\section{(6) OPEN ACCESS}

\title{
Needs must: living donor liver transplantation from an HIV-positive mother to her HIV-negative child in Johannesburg, South Africa
}

\author{
Harriet Rosanne Etheredge, ${ }^{\oplus 1,2}$ June Fabian, ${ }^{3,4}$ Mary Duncan, ${ }^{3}$ Francesca Conradie, ${ }^{4,5}$ \\ Caroline Tiemessen, ${ }^{6,7}$ Jean Botha ${ }^{3,8}$
}

'Wits University Donald Gordon Medical Centre, Johannesburg, Gauteng, South Africa

IInternal Medicine, University of the Witwatersrand School of Social Sciences, Johannesburg, South Africa

${ }^{3}$ Wits University Donald Gordon Medical Centre, Johannesburg, South Africa

${ }^{4}$ Internal Medicine, University of the Witwatersrand Faculty of Health Sciences, Johannesburg, Gauteng, South Africa

Clinical HIV Research Unit, Johannesburg, South Africa ${ }^{6}$ Centre for HIV and STIS, National Institute for Communicable Diseases, Johannesburg, South Africa ${ }^{7}$ University of the Witwatersrand Faculty of Health Sciences, Johannesburg, South Africa

${ }^{8}$ Surgery, University of the Witwatersrand Faculty of Health Sciences, Johannesburg, South Africa

\section{Correspondence to}

Dr Harriet Rosanne Etheredge, Wits University Donald Gordon Medical Centre, Johannesburg, Gauteng, South Africa; harriet.etheredge@mediclinic. co.za

Received 22 October 2018 Revised 17 February 2019 Accepted 11 March 2019

\section{ABSTRACT}

The world's first living donor liver transplant from an HIV-positive mother to her HIV-negative child, performed by our team in Johannesburg, South Africa (SA) in 2017, was necessitated by disease profile and health system challenges. In our country, we have a major shortage of donor organs, which compels us to consider innovative solutions to save lives. Simultaneously, the transition of the HIV pandemic, from a death sentence to a chronic illness with excellent survival on treatment required us to rethink our policies regarding HIV infection and living donor liver transplantation. Although HIV infection in the donor is internationally considered an absolute contraindication for transplant to an HIV-negative recipient, there have been a very small number of unintentional transplants from HIV-positive deceased donors to HIV-negative recipients. These transplant recipients do well on antiretroviral medication and their graft survival is not compromised. We have had a number of HIV-positive parents in our setting express a desire to be living liver donors for their critically ill children. Declining these parents as living donors has become increasingly unjustifiable given the very small deceased donor pool in SA; and because many of these parents are virally suppressed and would otherwise fulfil our eligibility criteria as living donors. This paper discusses the evolution of HIV and transplantation in SA, highlights some of the primary ethical considerations for us when embarking on this case and considers the new ethical issues that have arisen since we undertook this transplant.

\section{INTRODUCTION}

The world's first living donor liver transplant from an HIV-positive mother to her HIV-negative child, performed by our team in Johannesburg, South Africa (SA) in 2017, was necessitated by disease profile and health system challenges in our country. ${ }^{1}$ This paper details the context and the ethical reasoning behind our painstaking decision to proceed with this transplant.

\section{THE CASE}

(c) Author(s) (or their employer(s)) 2019. Re-use permitted under CC BY-NC. No commercial re-use. See rights and permissions. Published by BMJ.

To cite: Etheredge $H R$ Fabian J, Duncan $\mathrm{M}$, et al. $J$ Med Ethics

2019;45:287-290
A 13-month-old child, diagnosed with biliary atresia, was wait-listed for a deceased donor liver transplant at our centre in Johannesburg, SA. Prior to listing, the child had undergone a Kasai portoenterostomy procedure, but this failed to establish biliary drainage. ${ }^{2}$ While on the deceased donor waiting list the child suffered numerous life-threatening complications secondary to established liver cirrhosis. These necessitated hospitalisation and intensive care unit admission. Throughout this process, the child's mother requested consideration as a living donor. We initially dismissed this request as the mother was known to be HIV-positive. The policy in our living donor liver transplant programme has always excluded HIV-positive living donors because of the risk of transmission.

As the child's health deteriorated, it became clear that the living donor option, with the child's HIV-positive mother as the donor, was our only hope of saving the child's life. Due to SA's solid organ shortage, it was highly likely that the child would die before a deceased donor liver could be procured. The HIV-positive living donor option was only pursued after all other willing family members had been found ineligible for living donation. The child remained on our deceased donor waiting list until transplant, and at the time of transplant had been listed for 181 days, almost four times the average for our programme.

At the time of writing, 21 months after the procedure, the donor mother is fully recovered and remains in good health. The recipient child is thriving, with indeterminate-possibly negativeHIV serostatus. ${ }^{1}$

\section{THE CONTEXT}

\section{HIV and transplantation in SA}

Over the past 15 years, SA has emerged from a troubling era of AIDS denialism which resulted in the deaths of approximately 330000 people. In 2002, sustained civil society advocacy culminated in a court ruling that obligated the government to roll out antiretroviral therapy (ART). ${ }^{3}$ Today, SA has the highest number of incident HIV infections in any country (national prevalence of $12.6 \%- \pm 7$ million people) and approximately half are on treatment (3.4 million). Through successful implementation of our national policy for HIV, child and infant mortality has decreased by $25 \%$ with prevention of mother-to-child transmission. Average life expectancy of HIV-positive people increased from 56 years to 61 years in the period from 2009 to 2012 alone. $^{3}$

SA also has a long history of solid organ transplant that spans 50 years. Our health system has the depth and capacity to offer this highly specialised service. Compared with HIV management, access to transplantation is, unfortunately, less equitable and poorly funded-particularly in the state sector. ${ }^{4}$ 
Numerous factors are thought to influence solid organ availability in SA, and these seem to occur at many different levels simultaneously. In some cases, public perceptions of transplant and its portrayal in the media have damaged the image of organ donation —often creating uncertainty and mistrust—and this has discouraged people from donating. ${ }^{5}$ There are also challenges at the level of health facilities, where staff may hold unfavourable attitudes to organ transplant or where training and staffing in transplant specialities is not prioritised. ${ }^{6}$ Published research has also found a policy vacuum in transplant, where hospital staff do not understand what is required of them. This may influence transplant numbers and discourage staff from referring potential donors. $^{7}$

Wits Donald Gordon Medical Centre is a private academic teaching hospital in the Faculty of Health Sceinces, University of the Witwatersrand, Johannesburg. In response to an unmet need for liver transplantation, we started a paediatric liver transplant programme in 2004. To increase access to transplantation for children with liver failure, we expanded the donor pool by introducing a living donor liver transplant programme in 2013. ${ }^{46}$ As more children were referred to our programme for evaluation of end-stage liver disease, questions about the feasibility of transplantation from an HIV-positive living donor were frequently raised by our staff and by parents. HIV-positive status precluding parents from donation to their children was a cause of consternation.

HIV infection in the donor, when the intended recipient is HIV-negative, is an internationally accepted contraindication for both deceased and living donation and, in some countries it is illegal. ${ }^{8}$ However, there are documented case reports of inadvertent transmission of HIV to previously uninfected recipients through deceased donor solid organ transplantation ${ }^{10}$ and, we have first-hand experience of this in our own programme (unpublished). Given the circumstances of these inadvertent transmissions, there was no option to administer prophylaxis to prevent HIV seroconversion prior to the transplant procedure. It appears that with ART, overall outcomes and survival in recipients who received HIV-positive deceased donor organs and seroconverted is as good as those who received HIV-negative deceased donor organs, even without prophylactic measures. ${ }^{10}$

What does this mean for transplant practice and HIV in SA? Now more than ever, we have increasing numbers of HIV-positive individuals, with good virological suppression and well-preserved CD4 counts, who would be suitable living donors. This raised a number of questions for our transplant team: Was it really appropriate for us to continue denying the option of living liver donation to HIV-positive adults who expressed willingness to do so, and would otherwise be eligible? Was this a decision which adequately facilitated their autonomy and the best interests of their children? Were we doing our best to anticipate the growing transplant need for children with organ failure and their families in SA? As the nature of HIV had been reframed, we realised that our transplant programme had to be appropriately situated within this context.

\section{REGULATORY MATTERS AND INSTITUTIONAL REVIEW BOARD (IRB) APPROVAL}

Prior to the transplant, we undertook constructive engagement on the clinical and ethical issues with our Institutional Review Board (IRB). During this process, the IRB carefully considered the ethical issues highlighted later in the article, as well as the context-which suggested that this was the only option to save the life of the child in question. The IRB ultimately gave us authorisation to perform the case under the auspices of Section 37 of the 2013 Declaration of Helsinki. ${ }^{9}$ This section states the following:

In the treatment of an individual patient, where proven interventions do not exist or other known interventions have been ineffective, the physician, after seeking expert advice, with informed consent from the patient or a legally authorised representative, may use an unproven intervention if in the physician's judgement it offers hope of saving life, re-establishing health or alleviating suffering. This intervention should subsequently be made the object of research, designed to evaluate its safety and efficacy. In all cases, new information must be recorded and, where appropriate, made publicly available.

HIV-positive to HIV-negative living donor liver transplant has now been formalised as a research programme at Wits Donald Gordon Medical Centre (institutional ethics clearance \# M170290 and \#M171035) and this work has been published ${ }^{14}$ as per the stipulations of Section 37.

\section{ETHICAL ISSUES}

\section{Risk of HIV transmission versus the benefit of saving a life}

Navigating the ethical quandaries presented by this case was not straightforward. Given the serodiscordance of our donor and recipient, we did not have any data on which to base our analysis of risk for mother and child becuase a liver transplant with this HIV profile (HIV-positive living adult donor, HIV-negative recipient child) had not been undertaken before. Some aspects were obvious. This was a therapeutic intervention with the prospect of direct benefit to the recipient child-saving their life. The primary risk was the possibility that the intervention to save the recipient's life would also infect them with HIV. Given that HIV is imminently manageable,${ }^{11}$ and transplant recipients appear to tolerate ART and immunosuppression well, it was unanimously agreed that the immediate benefit outweighed the immediate risk. A further consideration was that we would be able to carefully control possible HIV transmission, by initiating ART prophylaxis in the recipient prior to the procedure, as well as selecting a long-term virally suppressed donor.

Arguments weighing the risk of HIV transmission with the benefit of saving a child's life have taken on new prescience subsequent to our initial publication of this transplant, when the issue was debated in a meeting to establish guidelines for HIV-positive to HIV-negative transplantation. HIV clinicians have argued that, in the context of living with a chronic illness, HIV is perhaps preferable to others such as diabetes mellitus or cancer, given the good survival and simplified treatment regimens. However, the stigma around HIV still persists, and that adds complexity to the ethical issues we faced. Also noteworthy is HIV experts' contention that failure to offer HIV-positive parents the option of donation to their HIV-negative children is an infringement of their autonomy and contrary to the best interests of their children.

Based on available literature, the surgical risk to the mother as an HIV-positive adult with a well-preserved CD4 count and undetectable viral load undergoing living donor hepatectomy was deemed to be no greater than that for other living liver donors. ${ }^{12}$ However, a potential donor with active, untreated HIV infection would be excluded from this programme. In the latter scenario, the HIV-infected donor faces a risk of postoperative complications and death that is higher than an HIV-negative living donor. This is because untreated HIV infection is associated with immune dysregulation and CD4 depletion, 
opportunistic infections-most commonly tuberculosis, and comorbid viral infections such as hepatitis B and C. ${ }^{13}$ In this instance, a strong argument against donation can be made, mostly to protect the donor, and to protect recipients who could potentially be at higher risk of HIV infection due to the donor viraemia, and other opportunistic infections.

\section{An uncertain future}

Although the risk/benefit analysis of HIV transmission in the face of certain death for the child without a transplant was relatively straightforward, we could not anticipate the nature of indirect future risks related to drug regimens and interactions, or graft rejection. Although our recipient is doing well, the future in this regard remains uncertain, as it does for those who have inadvertently received HIV-positive deceased donor organs. For us, the tangible direct benefit outweighed the risk of an uncertain future. There are, however, questions about how the child may cope facing numerous uncertainties growing up. If our child is HIV-positive, this may have implications for social interactions and future relationships. It may also influence decisions that the child makes getting older. This differs from the small group of transplant recipients who inadvertently received HIV-positive donor organs. These individuals know they are HIV-positive and will require ART in the future. In this case, we do not know if our recipient child is HIV-infected. This creates a dual uncertainty: for the recipient and family, and for the medical team. Until we have a better sense of the child's HIV status, the family will have to negotiate this uncertainty with us, and this could be stressful for all parties.

\section{Information giving, consent and best interests}

One important ethical feature of this case was that the child did not have a say in the decision to proceed with this transplant, being too young to provide meaningful consent. This is not unique to our case, where the decision fell to the parents. Parents routinely make medical decisions about management of diseases in their children, and the over-riding ethical principal that should guide the actions of health professionals is the best interests of the child. However, a well-documented phenomenon in living donor transplant is that parents will often assume excessive risks to themselves to save the lives of their children. ${ }^{14}$ The intangible quality of the bond between parent and child means that this instinctual response is usually intrinsic to human nature and it cannot be separated from the transplant process. However, it also casts some doubt about the extent to which the decision to become a living donor and potentially infect one's child with HIV is an autonomous one. This consideration influenced the way we communicated risks and benefits to the child's parents.

A core aspect of the information-giving and consent process was ensuring that the parents appreciated the risk to their child. We were acutely aware of entering unknown territory and went to great lengths to ensure appropriate and detailed communication. We emphasised that we were unsure whether the child would contract HIV. We also took care to ensure that both parents had the capacity and social support to care for an HIV-positive child in the future. Our independent donor advocate (IDA), who is a multilingual community social worker, played a vital role in this process. The parents had several preliminary meetings with the IDA, and post-transplant she continued to informally make representations to the transplant team on behalf of the parents if necessary. Although it will never be possible to remove the emotional ties that compel people to make decisions with an unquantifiable risk-like the decision our parents made in this case-the IDA assists parents in their deliberations and in considering their options from all angles.

Although engaging the services of an IDA in living donor transplantation is routine in many countries, this is not the case in SA. We feel that this mechanism was successful in providing an extra layer orfcomfort and protection for our parents as they went through the decision-making process-and that the involvement of the IDA assisted in promoting parental autonomy as far as possible. The IDA was also empowered to engage with the medical team, thus mitigating an often-large power-differential between medical team and patients to some extent. Although it may require additional resources, it seems that all living donor transplant programmes in SA should have access to an IDA to ensure high standards of ethical practice.

\section{Fairness and equity}

We endeavoured to position this transplant within an ethical framework that was responsive to our context, fair and equitable. We go some way towards achieving more equitable access to liver transplantation through our programme, which allocates and transplants livers based on need, regardless of payer status. ${ }^{4}$ Our programme is the first in SA to offer this level of access. Ability to pay for healthcare coverage is an important determinant of who ultimately receives treatment in $\mathrm{SA}$, and it is known to perpetuate socioeconomic inequalities.

\section{NEW ETHICAL ISSUES Disclosure and diagnosis}

Subsequent to the publication of our case report on this transplant, a number of new ethical issues have come to light that warrant careful consideration.

At the outset of this case, we moved forward on the basis that the recipient was going to seroconvert and become HIV-positive due to the transplant. However, at this stage it is unclear whether seroconversion has taken place or not. For a child growing up, this casts a new shadow of uncertainty that we were unable to anticipate. It also raises questions of disclosure, how to disclose and when to disclose. These questions remain unanswered but are especially important given that we may not know the HIV status of the child for some time. This situation begs the question of how and when to disclose an uncertainty, and the implications of doing so for the future management of the child going forward. Especially important is the autonomy of the child as they get older-and the obligation to include the child in decision-making as far as possible. With so much unknown, this may pose a particular challenge.

Another emerging ethical issue is the extent to which the team ought to seek a definitive HIV diagnosis in the child-and this requires a careful risk-benefit analysis. It has been suggested that a provocative discontinuation of ART may be the only way to know for certain. However, discontinuing ART in an HIV-positive individual comes with significant risk. Reactivation of viral replication might occur. This could be at any time point after cessation of ART, necessitating regular screening of the child for an unknown period of time. Reactivation of HIV replication as a consequence of interrupting ART, while on immunosuppressive therapy to prevent rejection, may have life-threatening complications. This risk needs to be weighed against the risk of keeping a person who may be HIV-negative on ART, which might be unnecessary. Although case studies have demonstrated that HIV-positive transplant recipients tolerate both immunosuppression and ART well, ART does have long-term side effects 
and it would be important to ensure that a person was only taking ART because it was indicated by HIV infection.

\section{THE WAY FORWARD}

HIV-positive living donor liver transplant to HIV-negative paediatric recipients pivots around a number of central questions: Is it ethical to save a child's life through transplant while at that same time knowingly exposing them to HIV? Is it ethical to place the burden of this decision on a parent who may stop at nothing to save the life of their child? Is it ethical to deny HIV-positive people the option of living donation even when they are otherwise eligible to donate? To definitely determine whether the child does or does not have HIV infection, is a provocative treatment interruption of ART ethically justifiable, given the potential consequences? We have interrogated and agonised over these questions in our setting, and we will be faced with difficult decisions in the future. However, while we actively seek answers, the success of our first case is reason for optimism.

Contributors HRE: All drafts and ethics work on the project. JF: All drafts. MD: Transplant coordinator and draft checking. FC: Draft checking. CT: HIV sciences and draft checking. JB: PI and surgeon, all drafts.

Funding The authors have not declared a specific grant for this research from any funding agency in the public, commercial or not-for-profit sectors.

Competing interests None declared.

Patient consent for publication Not required.

Ethics approval Human Research Ethics Committee (Medical), University of the Witwatersrand, Johannesburg.

Provenance and peer review Not commissioned; externally peer reviewed.

Data sharing statement There are no unpublished data relating to this study available.

Open access This is an open access article distributed in accordance with the Creative Commons Attribution Non Commercial (CC BY-NC 4.0) license, which permits others to distribute, remix, adapt, build upon this work non-commercially, and license their derivative works on different terms, provided the original work is properly cited, appropriate credit is given, any changes made indicated, and the use is non-commercial. See: http://creativecommons.org/licenses/by-nc/4.0/.

\section{REFERENCES}

1 Botha J, Conradie F, Etheredge $\mathrm{H}$, et al. Living donor liver transplant from an HIVpositive mother to her HIV-negative child: opening up new therapeutic options. AIDS 2018;32:F13-19.

2 Esteves E, Clemente Neto E, Ottaiano Neto M, et al. Laparoscopic Kasai portoenterostomy for biliary atresia. Pediatr Surg Int 2002;18:737-40.

3 Simelela N, Venter WD, Pillay Y, et al. A political and social history of HIV in South Africa. Curr HIVIAIDS Rep 2015:12:256-61.

4 Tager S, Etheredge HR, Fabian J, et al. Reimagining liver transplantation in South Africa: a model for justice, equity and capacity building - the wits donald gordon medical centre experience. S Afr Med J 2019;109:84-8.

5 Etheredge H, Penn C, Watermeyer J. Opt-in or opt-out to increase organ donation in South Africa? Appraising proposed strategies using an empirical ethics analysis. Dev World Bioeth 2018;18:119-25.

6 Etheredge HR, Penn C, Watermeyer J. Interprofessional communication in organ transplantation in gauteng province, South Africa. S Afr Med J 2017;107:615-20.

7 Crymble K, Etheredge HR, Fabian J, et al. Nurses' knowledge about and attitudes toward organ donation in state and private hospitals in Johannesburg, South Africa. Southern African Journal of Critical Care 2017:33:52-7.

8 Burra P. European Association for the Study of the Liver. Electronic address: easloffice@easloffice.eu.EASL clinical practice guidelines: liver transplantation. J Hepatol 2016;64:433-85.

9 Transplantation Society of Australia and New Zealand. Clinical guidelines for organ transplantation from deceased donors. 2016 https://www.tsanz.com.au/organallocat ionguidelines/ (Accessed 19th Oct 2018).

10 American Transplant Congress. Outcomes of solid organ transplantation from an hiv positive donor to negative recipients. $2016 \mathrm{https}$ ://atcmeetingabstracts.com/abstract/ outcomes-of-solid-organ-transplantation-from-an-hiv-positive-donor-to-negativerecipients/ (Accessed 19th October 2018)

11 Teeraananchai S, Kerr SJ, Amin J, et al. Life expectancy of HIV-positive people after starting combination antiretroviral therapy: a meta-analysis. HIV Med 2017:18:256-66

12 Horberg MA, Hurley LB, Klein DB, et al. Surgical outcomes in human immunodeficiency virus-infected patients in the era of highly active antiretroviral therapy. Arch Surg 2006;141:1238-45.

13 Shanthamurthy D, Manesh A, Zacchaeus NG, et al. Perioperative outcomes in human immunodeficiency virus-infected patients - the PRO HIV study. Int J STD AIDS 2018;29:968-73.

14 Kruper A, Zanowski SC. Parental live liver donation: psychosocial considerations in the decision to donate. Curr Opin Organ Transplant 2015;20:140-5. 